\title{
Disk Weather
}

\section{Baroclinic Instability and Vortex Amplification}

\author{
Hubert Klahr ${ }^{1, a}$, Natalie Raettig ${ }^{1, b}$, and Wlad Lyra ${ }^{2, c}$ \\ ${ }^{1}$ Max-Planck Institute für Astronomie, Königstuhl 17, 69117 Heidelberg, Germany \\ ${ }^{2}$ Jet Propulsion Laboratory - California Institute of Technology, 4800 Oak Grove Drive, Pasadena CA 91109, \\ USA
}

\begin{abstract}
Recent years have shown that accretion disks around young stars have extended regions, which are too low ionized to couple to magnetic fields and thus the nature of the underlying turbulence cannot be exclusively magnetic. We also found that disks have in general a baroclinic density and temperature structure which means that a typical disk is radially buoyant and has a vertical velocity gradient also known as thermal wind. Here we show that the expected entropy gradients in observed accretion disks around young stars are in fact steep enough and that the thermal relaxation times are sufficiently short to allow for efficient amplification of vortices.
\end{abstract}

\section{Introduction}

Klahr and Bodenheimer (2003) [1] introduced the idea that a radial entropy gradient or more precisely a negative square of the radial Brunt-Väisälä frequency $N^{2}$ can destabilize a Rayleigh stable accretion disk. They had performed 3D radiation hydro simulations of global chunks of convection in accretion disks with vertical and radial entropy gradients and observed the formation of vortices and waves. They referred to this phenomenon as a "Global Baroclinic Instability" (GBI), because it is the global radial entropy gradient which gives the baroclinic term $(\nabla \Sigma \times \nabla P)$ in the vorticity equation the possibility to obtain non-zero values for non-axissymetric entropy distributions. This baroclinic term drives both the generation of net potential vorticity in the disk and gives the onset for a turbulent wave pattern. Nevertheless Klahr (2004) [2] showed that there is no linear instability for baroclinic disks. Finally Petersen et al. (2007a,b) [3,4] showed in their global incompressible simulations that in addition to radial entropy gradients a disk needs the right amount of thermal readjustment in order to let vortices grow. The combined condition of strong enough perturbations and sufficiently large Reynolds numbers led them to the conclusion that the Baroclinic Instability in accretions disks is actually a non-linear feature.

The radial temperature structure and thus the radial entropy and pressure stratification are determined by the combined influence of stellar irradiation, internal viscous heating and internal radiative heat transport via radiation. If the heat transport in such a baroclinic disk has an effective thermal

\footnotetext{
ae-mail: klahr@mpia.de

be-mail: raettig@mpia.de

ce-mail: Wladimir.Lyra@jpl.nasa.gov
}

This is an Open Access article distributed under the terms of the Creative Commons Attribution License 2.0, which permits unrestricted use, distribution, and reproduction in any medium, provided the original work is properly cited. 


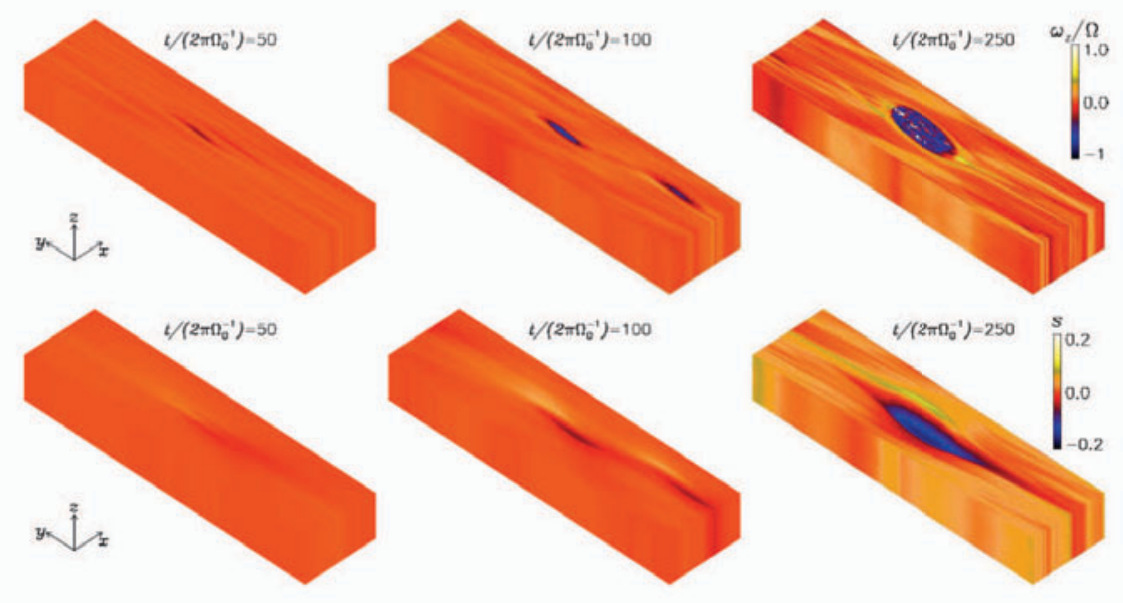

Figure 1. Evolution of vorticity (upper panels) and entropy (lower panels) due to the baroclinic instability in 3D. $t /(2 \pi \Omega)$ measures the time in number of orbits; $\omega_{z} / \Omega$ is the vertical component $(z)$ of the vorticity in units of the Keplerian frequency, $s$ is the specific entropy of the fluid; $x$ and $y$ are radial and azimuthal direction in the Keplerian shear flow of an accretion disk.

As time evolves from left to right a vortex with negative vorticity (blue) growth in the background of positive vorticity (red). The growth is linked to a local entropy perturbation (yellow higher, blue lower entropy), which is a source of vorticity via the Baroclinic term in the vorticity equation.

relaxation time of $0.1-100$ orbits, then a so called Subcritical Baroclinic Instability (SBI) will amplify small vortices to giant anti-cyclons (see Fig. 1) as has been shown in local 3D shearing sheat simulations by Lesur and Papaloizou (2000) [5] and Lyra and Klahr (2011) [6]. These vortices will have strong influence on disk evolution and on planet formation via the efficient concentration of dust.

This paper is organized as follows: In Section 2 we discuss realistic properties of disks around young stars, deriving radial Richardson Numbers and thermal relaxation times. In Section 3 we present numerical simulations on vortex growth for the parameter range presented in Section 2. Section 4 summarizes the current understanding on Stability and Vortex Growth in disks around young stars and its implications on the planet formation within.

\section{How radially buoyant are Circumstellar disks?}

Andrews et al. (2009) [7] used observed radial density profiles and modeled them with viscous accretion disk models, e.g. by calculating an $\alpha$ viscosity value for the measured mass accretion rate. Using their best fits to the radial surface density profiles

$$
\beta_{\Sigma}=\frac{d \log \Sigma}{d \log r}=0.9 \pm 0.2
$$

we can already estimate that the viscosity profile is $v \propto r^{\beta_{\Sigma}}$ because a steady state accretion disk has to possess a radially constant accretion rate $\partial_{r} \dot{M}=\partial_{r} \Sigma v=0$. Using the $\alpha$ ansatz, $v=\alpha c_{s}^{2} / \Omega$ determines the radial temperature gradient to be

$$
\beta_{T}=\frac{d \log T}{d \log r}=1.5 \quad \beta_{\Sigma}=0.6 \pm 0.2
$$



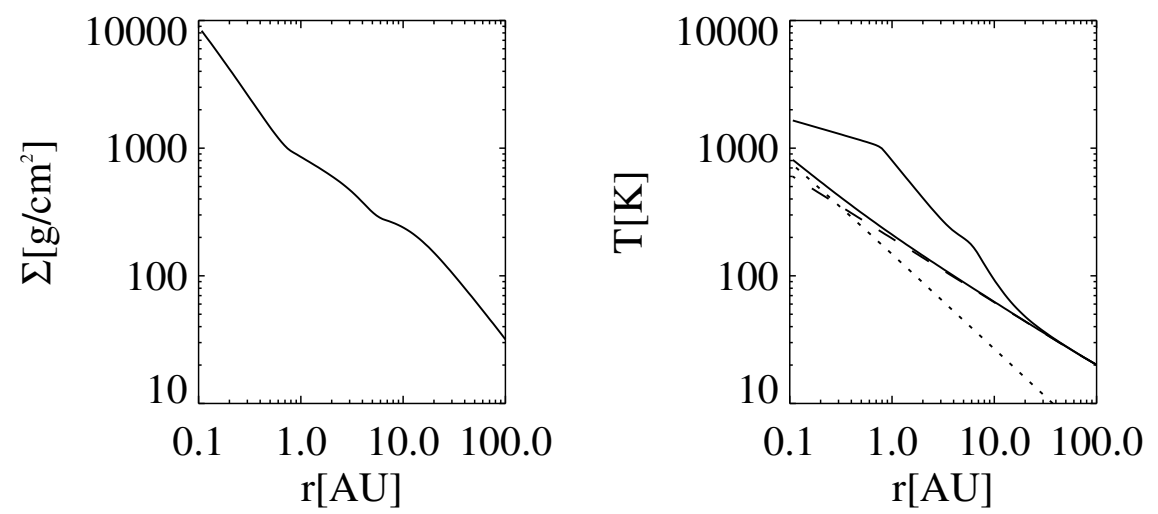

Figure 2. Temperature and surface density structure of a protoplanetary accretion disk with an accretion rate of $\dot{M}=1 \times 10^{-7} M_{\odot} / \mathrm{yr}$, an assumed efficiency of turbulent diffusion of angular momentum of $\alpha=3 \times 10^{-3}$ around a T-Tauri star with $T_{\text {eff }}=4300 K$ and $R_{\star}=2 R_{\odot}$. The dashed line is the surface temperature if the disk was only heated by stellar radiation and the dashed line shows the surface temperature if the disk was only heated up via viscous heating. these two processes make up the effective surface temperature (lower solid line). The upper solid line shows the derived mid-plane temperature.

for a constant $\alpha$. It might seem as a surprise that the pressure has a constant slope of

$$
\beta_{p}=-\frac{d \log p}{d \log r}=1.5
$$

independent of the radial density profile, but this is a consequence of constant accretion rate and the original hypothesis that viscous stresses are proportional to the local pressure. The entropy gradient can now be derived from the temperature and surface density gradients $\beta_{K}=\beta_{T}-(\gamma-1) \beta_{\Sigma}$ as

$$
\beta_{K}=-\frac{d \log K}{d \log R}=1.5-\gamma \beta_{\Sigma}=0.28 \pm 0.27,
$$

with $K$ as a potential temperature like representative for entropy following the polytropic equation of state $p=K \Sigma^{\gamma}$ with $\gamma=1.353$ the $2 \mathrm{D}$ adiabatic index. These values are always positive, indicating the unstable regime of entropy gradients, violating the radial Schwarzschild criterion as expressed by negative values for the square of the Brunt-Väisälä frequency $N^{2}<0$.

We extended these estimates by adopting irradiated vertical atmosphere models of accreting disks (see Fig. 2) and were thus able to derive the radial value of the Richardson number and the local thermal relaxation time as a function of radius (see Fig. 3).

\section{Is the radial buoyancy sufficient for vortex amplification?}

Recent studies [8] have shown that baroclinic vortex amplification is strongly dependent on the global entropy gradient, the efficiency of thermal diffusion and/or relaxation as well as on the numerical resolution. We conducted a comprehensive study of a broad range and combination of various entropy 

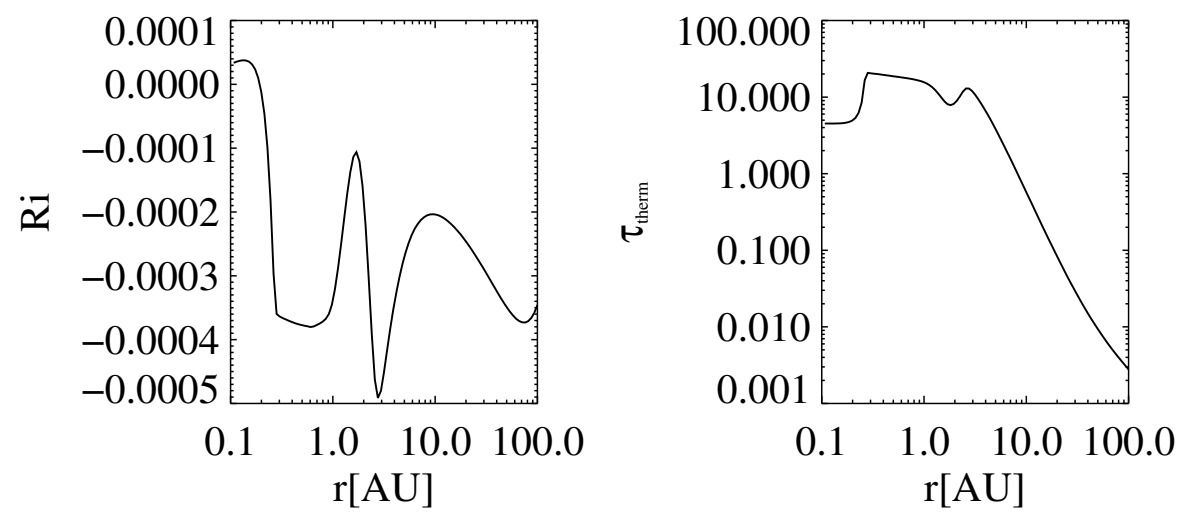

Figure 3. Richardson number and thermal diffusivity in a protoplanetary accretion disk with an accretion rate of $\dot{M}=1 \times 10^{-8} M_{\odot} / \mathrm{yr}$, an assumed efficiency of turbulent diffusion of angular momentum of $\alpha=3 \times 10^{-3}$ around a T-Tauri star with $T_{\text {eff }}=4300 \mathrm{~K}$ and $R_{\star}=2 R_{\odot}$.

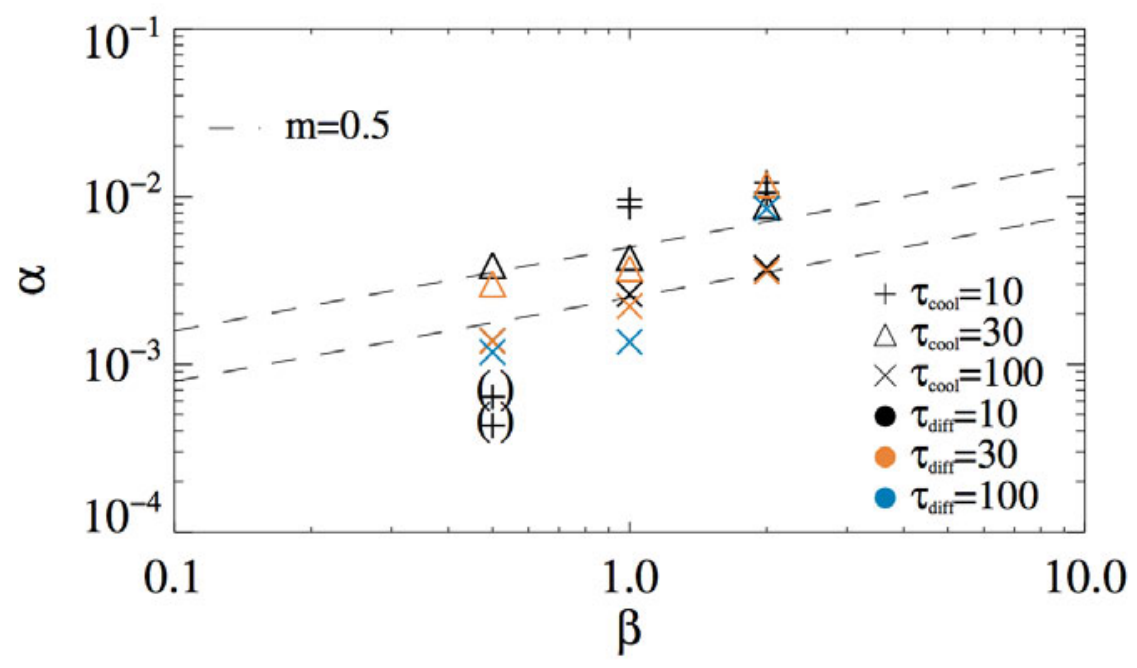

Figure 4. Saturation values of baroclinic turbulence measured in Reynolds stress parameter $\alpha$ depending on the radial entropy and pressure gradient $\beta$. The symbols show the different combinations of thermal relaxation processes with their characteristic timescales: cooling via the photosphere (line shape of symbol) and thermal diffusion within the plane of the disk (color of symbol). 
gradients, thermal diffusion and thermal relaxation time-scales via local shearing sheet simulations covering the parameter space relevant for protoplanetary disks.

We measure the Reynolds stresses as a function of our control parameters (see Fig. 4) and see that there is angular momentum transport even for effective entropy/pressure gradients as low as $\beta=$ $\sqrt{\beta_{K} \beta_{P}}=0.5$ The amplification-rate of the perturbations, appears to be proportional to $\beta^{2}$ and thus proportional to the square of the Brunt-Väisälä frequency $N^{2}$. Migration of the vortex appears to be a lesser problem as the vortices are too small for effective radial drift for most of their growth time. The saturation level of Reynolds stresses on the other hand seems to be proportional to $\beta^{\frac{1}{2}}$. This highlights the importance of baroclinic effects even for the low entropy gradients expected in protoplanetary disks.

\section{Conclusions}

Observed disks around young stars appear to have the right radial entropy structure and are in the right thermal relaxation regime to allow for efficient amplification of vortices.

We currently investigate the birth of the first vortices in vertically stratified disks and also study the concentration of dust in anti-cyclons. Here we found a very fast triggering of the streaming instability even in very low metallicity which is the onset of planetesimal formation.

\section{References}

[1] Klahr, H., \& Bodenheimer, P., Astrophysical Journal 582, 869-892 (2003)

[2] Klahr, H., Astrophysical Journal 606, 1070-1082 (2004)

[3] Petersen, M. R., Julien, K., \& Stewart, G. R., Astrophysical Journal 658, 1236 (2007)

[4] Petersen, M. R., Stewart, G. R., \& Julien, K., Astrophysical Journal 658, 1252 (2007)

[5] Lesur, G., \& Papaloizou, J. C. B., Astronomy and Astrophysics 513, A60 (2010)

[6] Lyra, W., \& Klahr, H., Astronomy and Astrophysics 527, A138 (2011)

[7] Andrews, S. M., Wilner, D. J., Hughes, A. M., Qi, C., \& Dullemond, C. P., Astrophysical Journal 700, 1502 (2009)

[8] Raettig, N., Klahr H., \& Lyra, W., Astrophysical Journal submitted, arXiv:1212.4464v1 (2013) 